\title{
Mathematical fitting for the variation in capacity of lithium iron phosphate batteries corresponding to cycles
Chi-Yao Chung ${ }^{1}$
Kung-Yen Lee ${ }^{2}$
Ting-Jung $\mathrm{Kuo}^{3}$
Zih-Yeh $\operatorname{Lin}^{4}$
Shuen-De $\mathrm{Wu}^{5}$
Chiao $\mathrm{Fu}^{6}$

(Received 9 January 2016; revised 13 October 2016)

\begin{abstract}
The capacity of lithium iron phosphate $\left(\mathrm{LiFePO}_{4}\right)$ batteries decreases as the usage cycles increase. In order to investigate the relationship between the number of charge/discharge cycles and the battery capacity, two batteries were cyclically charged and discharged using an automatic testing system, while simultaneously collecting the capacity data. The tested batteries were composed of different materials and had a capacity of $15 \mathrm{Ah}$. Each battery was cyclically charged and discharged 800 times. The batteries are analyzed and compared with each other to create fitted curves. The developed mathematical fitting,
\end{abstract}

DOI:10.21914/anziamj.v57i0.10436, (C) Austral. Mathematical Soc. 2016. Published October 26, 2016, as part of the Proceedings of the 12th Biennial Engineering Mathematics and Applications Conference. ISSN 1445-8810. (Print two pages per sheet of paper.) Copies of this article must not be made otherwise available on the internet; instead link directly to the DOI for this article. Record comments on this article via http://journal. austms.org. au/ojs/index.php/ANZIAMJ/comment/add/10436/0 
which consists of both exponential and polynomial terms, is closely responsive to the battery capacity.

\section{Contents}

1 Introduction

C292

2 Experiment

C293

3 Results and discussion

C295

4 Conclusion

C301

References

C302

\section{Introduction}

Lithium iron phosphate $\left(\mathrm{LiFePO}_{4}\right)$ batteries have a variety of superior properties compared to more common lithium cobalt oxide batteries, such as higher power densities, higher capacities, longer lifetimes and better safety. For these reasons, $\mathrm{LiFePO}_{4}$ batteries are used extensively in electric vehicles, hybrid electric vehicles, and energy storage devices [1, 2]. However, there is an issue with the battery capacity in that it begins to rapidly decay after a certain number of charge and discharge cycles, which may cause safety problems. Therefore, it is very important to investigate the electrical characteristics (voltage, current, and capacity) of $\mathrm{LiFePO}_{4}$ batteries in relation to the number of cycles [3, 4, 5, 6, 7].

Because of electrochemical reactions and the aging of the material within the battery, it is difficult to determine the remaining capacity of a battery with respect to the number of cycles $[3,4,8,9]$. Hence, the aim of this study is to validate the proposed mathematical fitting by comparing measurement results 
with fitted results. The proposed model contains both an exponential term and a polynomial term. The parameters of the two terms are obtained by fitting the capacity variation curve of the $\mathrm{LiFePO}_{4}$ batteries. The exponential term represents the active electrochemical reaction during the initial usage stage of a new battery. The polynomial term represents the steady state of the battery. The capacity of the battery gradually decreases in the steady state.

\section{Experiment}

A model for $\mathrm{LiFePO}_{4}$ batteries is used to simulate the behavior of the batteries. The battery model is an open-circuit voltage source connected to a single series resistor and a single resistor-capacitor (RC) network. The capacity is regarded as a nonlinear capacitor so that it simulates the capacity conditions.

According to Faraday's Law, the battery capacity is equal to the integral of current over time. In the charging and discharging experiments, the current is constant so that the battery capacity is determined using the product of the current and the time. Therefore, the variation in capacity after a single charge/discharge cycle is obtained.

Figure 1 plots the experimental discharging voltage curves for new, 300cycle and 1000-cycle batteries for the constant-current (CC) and constantvoltage (CV) charging method and constant-current discharging method [10]. The figure indicates that the discharge time or the capacity of a battery declines with an increasing number of cycles.

Two types of $\mathrm{LiFePO}_{4}$ batteries composed of different materials are measured and compared. Each battery has the same capacity of $15 \mathrm{Ah}$ as well as the same number of cycles (800). At the beginning of the experiment, each battery was charged using a DC power generator with the CC-CV method. The battery was charged with the $\mathrm{CC}$ in the initial stage because a higher charging current might cause permanent damage to the battery. When the battery voltage reached the upper limit of the charging voltage, charging switched to the $\mathrm{CV}$ 
Figure 1: The discharging voltage curves for new, 300-cycle, and 1000-cycle batteries.

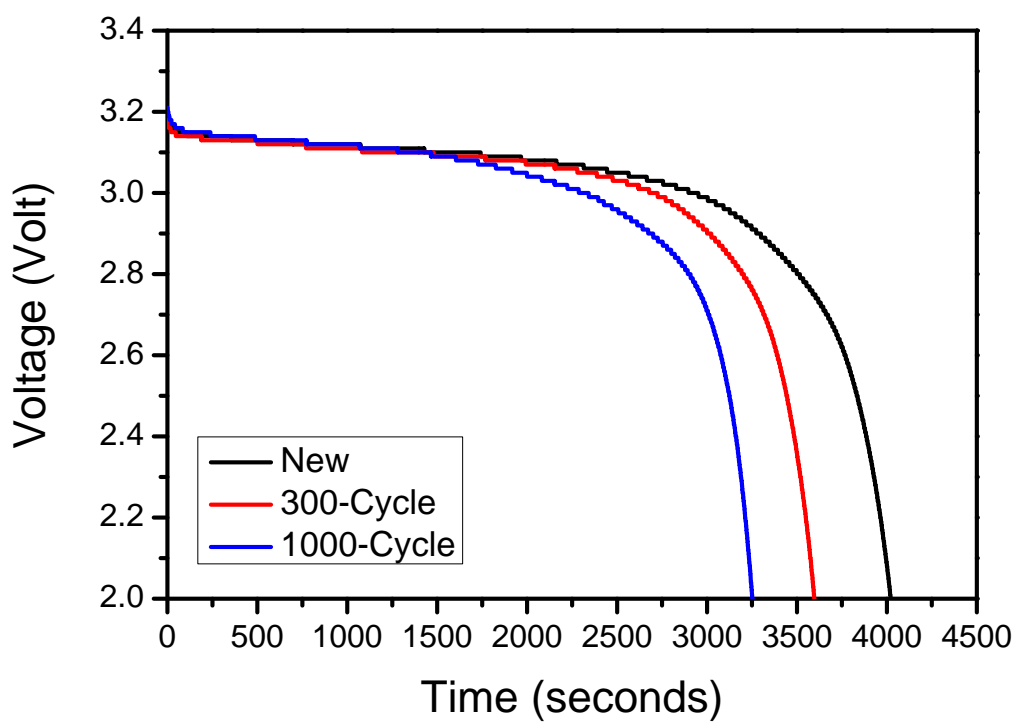

mode with a small current. This CC-CV method protects the battery from damage and over-charging during the charge process. It also reduces the charge time. The charging current rate and the upper limit charging voltage were set at $1 \mathrm{C}$ and $3.65 \mathrm{~V}$, respectively. In the charge process, different charging rates were not considered.

After the charging procedure, the battery was left idle until it was in a steady state, and therefore there is no significant difference in the initial voltages of the tested batteries, as shown in Figure 1. The batteries were then discharged using a discharging current rate of $1 \mathrm{C}$ through a DC electronic load. The $1 \mathrm{C}$ discharge rate means that it takes one hour to fully discharge a $15 \mathrm{Ah}$ battery with a discharging current of $15 \mathrm{~A}$. The cut-off voltage was set at $2.0 \mathrm{~V}$ since there is a significant difference in the lithium transfer reaction and irreversible damage can occur when the voltage falls below $2.0 \mathrm{~V}[11,12,13,14]$. During 
the discharging period, the battery voltage was automatically checked by a computer. If the voltage reached the cut-off voltage, then the discharging process was stopped. If not, then the process continued until the voltage fell to $2.0 \mathrm{~V}$. The capacity data was automatically recorded and collected by a computer. The tested batteries were cyclically charged and discharged 800 times. Consequently, the capacity of all batteries is determined from the integral of the discharging current over time.

A discharging current of $20 \mathrm{~A}$ was used in earlier experiments [10]. The temperature and resistance increase with this higher current, and the battery voltage decreases due to higher resistance, but the capacity of a battery is almost the same [10]. For the discharging current of $20 \mathrm{~A}$, the trend of the capacity loss is almost the same as the curve shown in Figure 2 for the discharging current of $15 \mathrm{~A}$. However, the slope is steeper and the discharge time is shorter for the $20 \mathrm{~A}$ case. If the temperature is too high, then the capacity of a battery is reduced. This is because the higher thermal resistance makes the voltage drop larger so that the battery voltage easily reaches the cut-off voltage during the discharge process, and then the total discharge time is shorter, leading to a smaller capacity.

\section{Results and discussion}

The experimental results and linear fitted curves for two types of battery, Type-A and Type-B, are shown in Figure 2(a) and Figure 2(b), respectively. The solid lines represent the experimental data and the dashed lines represent the linear fitted curves. The results show that the battery capacities vary depending on the type of battery. The capacities of the tested batteries reduce as the number of cycles increases. To identify the best fitted equation for the batteries, at first, two straight lines with negative slopes are programmed to fit the real data for Type-A and Type-B batteries from about 100 cycles to 800 cycles. The linear fitted curves are then extrapolated to the initial point (i.e., the number of cycles $=0$ ). The divergences of the real data from the 
Figure 2: The capacity variation and linear fitted curves for (a) Type-A and (b) Type-B batteries.
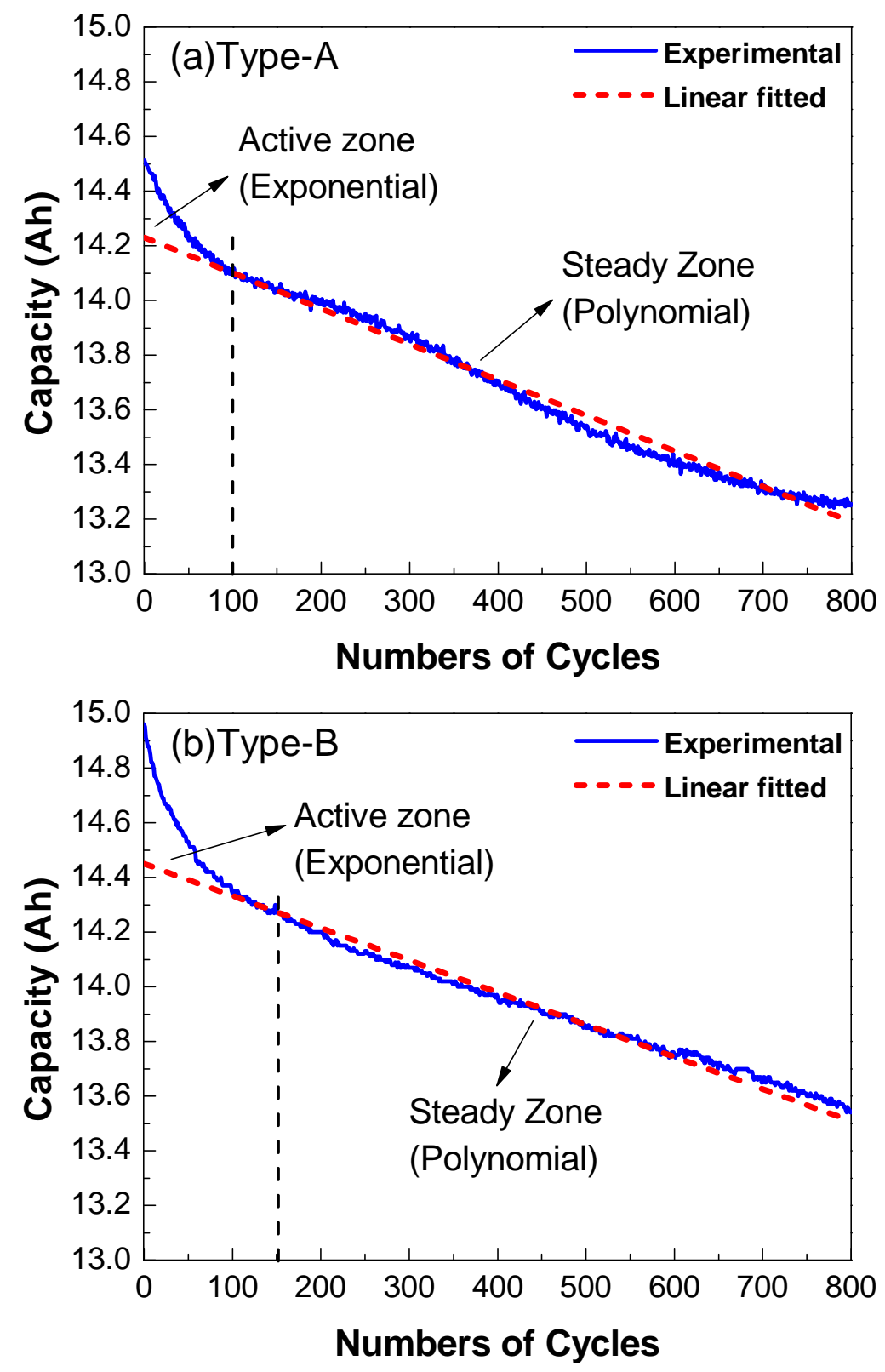
linear fitted curves for Type-A and Type-B batteries are at cycles 100 and 150, respectively (Figure 2). These divergences separate the experimental curves into two parts. The first part is the active zone, during which the capacity is unstable and decays exponentially; the second part is the steady zone, during which there is a slow, linear decay in capacity. From Figure 2, we see that a linear equation is unsuitable for the tested batteries when the capacity of the battery is in the active zone.

From the experimental curves of Figure 2, the drop in capacity in the active zone acts in a similar manner to the discharge behavior of an RC network [6]. Hence, the variation in capacity of the battery is a function of the number of cycles and the fitted equation is

$$
c(x)=a e^{-b x}+s x+i,
$$

where $x$ is the number of cycles, $a$ and $i$ are capacities, $a+i$ is the initial capacity $(x=0), b$ is the decay constant, and $s$ is the linear decay rate in the stable zone.

Equation (1) represents an equivalent electrical circuit which is composed of a nonlinear open-circuit voltage source, a series resistor and one resistercapacitor (RC) network [10]. The nonlinear open-circuit voltage source represents the open voltage of a battery. In equation (1) $a e^{-b x}$ represents one $\mathrm{RC}$ network because the charge and discharge behaviors of a capacitor is described by an exponential term, and the $s x+i$ term represents a linear resistor.

Figure 3 shows there are no significant differences between the fitted curves (dashed lines) and the experimental data curves (solid lines), indicating that the proposed fitted equation (1) closely matches the individual data for these two batteries. The exponential part dominates the rapid drop in capacity when the battery is in the active zone. As the number of cycles increases, the polynomial term dominates the decay rate of the capacity for the battery in the steady zone.

Although Figure 4 only shows up to 800 cycles of charging and discharging, the 
Figure 3: The fitted and experimental curves for (a) Type-A and (b) Type-B batteries.
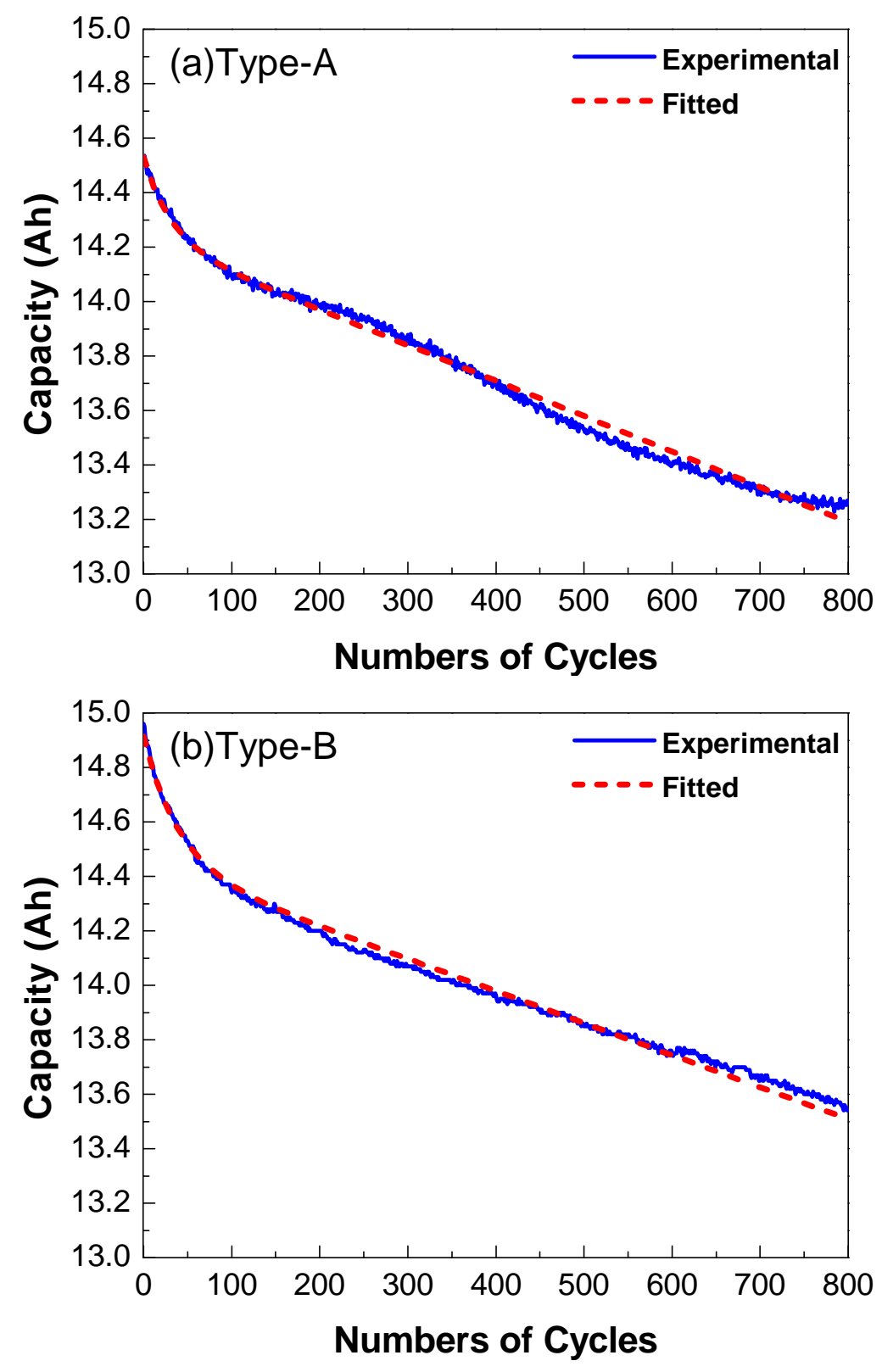
Table 1: The parameters of the mathematical fitting. Batteries

Parameters

\begin{tabular}{ccccc} 
& $\mathrm{a}(\mathrm{Ah})$ & $\mathrm{b}(1 /$ number $)$ & $\mathrm{s}(\mathrm{Ah} /$ number $)$ & $i(\mathrm{Ah})$ \\
\hline Type-A & 0.302 & 0.0319 & $-1.302 \cdot 10^{-3}$ & 14.23 \\
Type-B & 0.463 & 0.0254 & $-1.178 \cdot 10^{-3}$ & 14.45
\end{tabular}

fitted curves should be feasible as long as the battery continues to operated in the steady zone (e.g., up to 2000 cycles) thanks to the stability of an $\mathrm{LiFePO}_{4}$ battery.

Table 1 provides a summary of the parameters used in equation (1) for Type-A and Type-B batteries. The values for the two types of batteries are similar. The $\boldsymbol{a}$ and $i$ values for the Type-B battery are larger than the $a$ and $i$ values for the Type-A battery because the initial capacity of the Type- $\mathrm{B}$ battery is larger than that of the Type-A battery. The value of $b$ for the Type-B battery is less than the value of $b$ for the Type-A battery because the duration of the active zone for the Type-B battery is longer than that of the Type-A battery. The linear decay rates $s$ are very close for both batteries.

Figure 4(a) shows the experimental data for both the Type-A and Type-B batteries. The trends for the two types of battery are very similar. The difference in capacity between the Type-A and Type-B batteries remains almost the same, at around 0.4 Ah, as shown in Figure 4(b). The major difference between the two batteries is the initial capacity. The initial capacity of the Type-B battery reaches $15.0 \mathrm{Ah}$ at cycle zero. However, the duration of the active zone for the Type-B battery is slightly longer than that of the Type-A battery. The capacity at the boundary between the active zone and the steady zone is about $14.1 \mathrm{Ah}$ and $14.3 \mathrm{Ah}$ for the Type-A and Type-B batteries, respectively.

To calculate the accuracy of the fitted equation for the Type-A and Type-B batteries we use the mean absolute percentage error between the experimental 
Figure 4: (a) The experimental curves for the Type-A and Type-B batteries and (b) the difference in capacity between the Type-A and Type-B batteries.
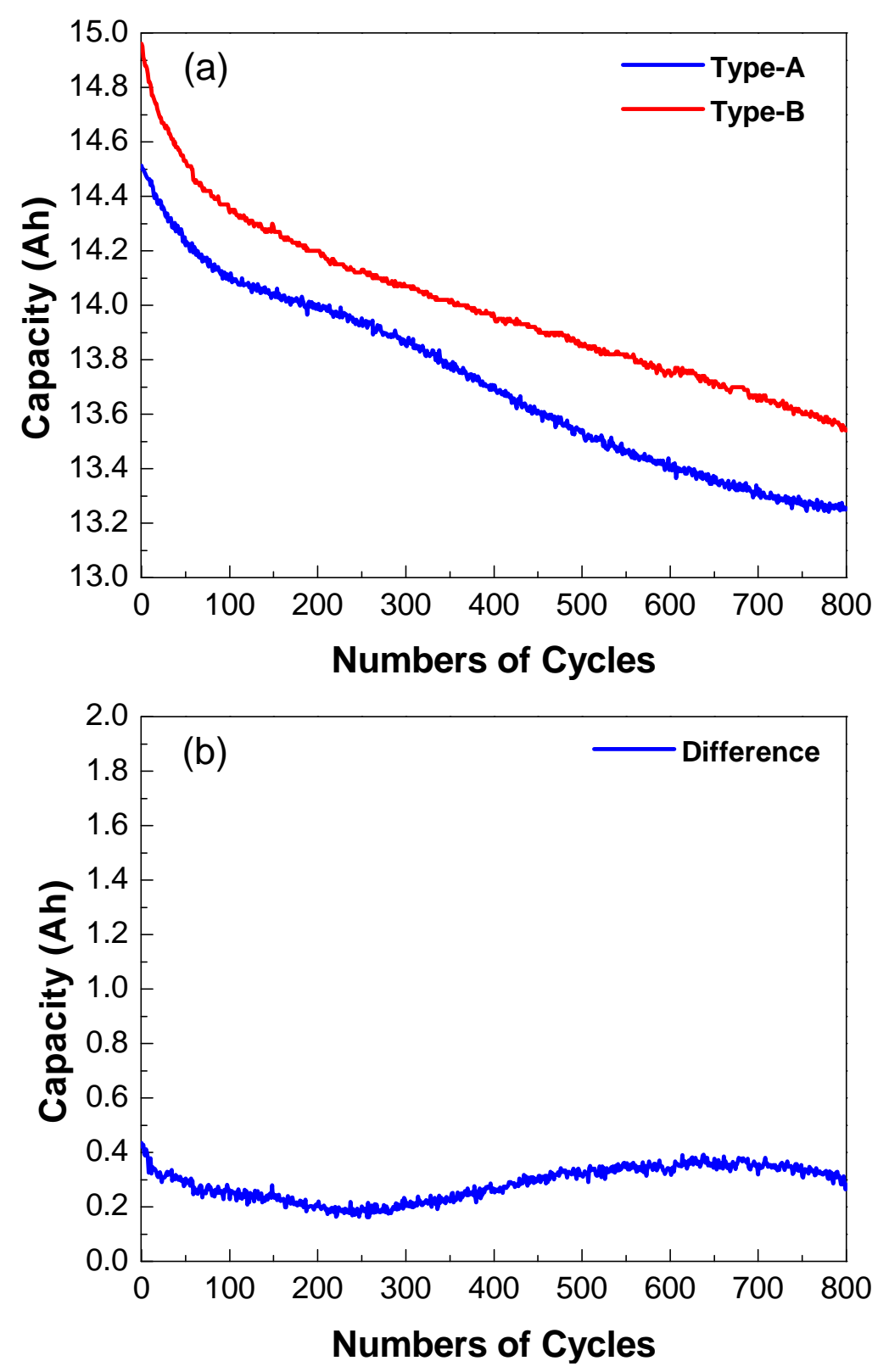
Table 2: The MAPE and error values for the two tested batteries.

\begin{tabular}{cccc} 
Category & MAPE $(\%)$ & Maximum error $(\%)$ & Minimum error $(\%)$ \\
\hline Type-A & 0.18 & 0.55 & 0.00068 \\
Type-B & 0.16 & 0.41 & 0.00042
\end{tabular}

data and the fitted curve

$$
\operatorname{MAPE}(\%)=\frac{1}{\mathrm{~N}} \sum \frac{\left|\mathrm{Q}_{\text {real }}-\mathrm{Q}_{\text {fit }}\right|}{\mathrm{Q}_{\text {real }}} \times 100 \%,
$$

where $\mathrm{Q}_{\text {real }}$ is the experimental data, $\mathrm{Q}_{\text {fit }}$ is the fitted curve, $\mathrm{N}$ is the total amount of data recorded for 800 cycles, and the sum is over these 800 data points.

Table 2 shows the MAPE value between the experimental data and the fitted curve is less than $0.18 \%$, and the maximum error is less than $0.55 \%$. Therefore, the fitted curve closely describes the relationship between the battery capacity and the number of cycles. In addition, once the battery capacity goes beyond the steady state, the capacity starts to rapidly decay. Equations (1) and (2) are used to analyze the capacity conditions of the battery. If the number of cycles passes more than 2000, and the error rate is much higher than the maximum error and continues to increase as the number of cycles increases, then it is an indication that either the limits of the battery capacity have been reached, or that the battery is fully discharged, meaning that the battery should be charged.

\section{Conclusion}

In this study, by separating the exponential and polynomial parts of the fitted equation, we are able to distinguish variations in the capacity of $\mathrm{LiFePO}_{4}$ batteries corresponding to the different numbers of charge and discharge cycles. When the battery is in the active state, the capacity decays exponentially, 
meaning that the exponential term in equation (1) dominates the capacity behavior. Once the battery reaches the steady zone, it shows a linear decay in capacity and the polynomial term is dominant. An analysis of the MAPE for the tested batteries shows that it is less than $0.18 \%$, meaning that this fitted curve is closely responsive to the capacity data for the batteries when the number of cycles is in the range of 0 to 800 . Furthermore, if the cycle number is beyond 800 , then equations (1) and (2) can be used to analyze the capacity conditions.

Acknowledgements The authors acknowledge the support of Ministry of Science and Technology Taiwan under Grants MOST 104-2221-E-002-108, 104-3113-E-002-004 and 104-3113-E-002-020-CC2.

\section{References}

[1] W. Su, H. Rahimi-Eichi, W. Zeng and M.-Y. Chow. A survey on the electrification of transportation in a smart grid environment. IEEE Trans. Ind. Inform., 8:1-10, 2012. doi:10.1109/TII.2011.2172454 C292

[2] J. Wang, Z. Sun and X. Wei. Performance and characteristic research in $\mathrm{LiFePO}_{4}$ battery for electric vehicle applications. IEEE Proc. Vehicle Power Propul. Conf., 1657-1661, 2009. doi:10.1109/VPPC.2009.5289664 C292

[3] T. Kim and W. Qiao. A hybrid battery model capable of capturing dynamic circuit characteristics and nonlinear capacity effects. IEEE Trans. Energy Conv., 26:1172-1180, 2011. doi:10.1109/TEC.2011.2167014 C292

[4] A. Eddahech, O. Briat and J.-M. Vinassa. Determination of lithium-ion battery state-of-health based on constant-voltage charge phase. J. Power Sources, 258:218-227, 2014. doi:10.1016/j.jpowsour.2014.02.020 C292 
[5] A. Shafiei, A. Momeni and S. S. Williamson. Battery modeling approaches and management techniques for plug-in hybrid electric vehicles. IEEE Proc. Vehicle Power Propul. Conf., 1-5, 2011. doi:10.1109/VPPC.2011.6043191 C292

[6] M. Chen and G. A. Rincon-Mora. Accurate electrical battery model capable of predicting runtime and i-v performance. IEEE Trans. Energy Conv., 21:504-511, 2006. doi:10.1109/TEC.2006.874229 C292, C297

[7] L. Gao, S. Liu and R. A. Dougal. Dynamic lithium-ion battery model for system simulation. IEEE Trans. Comp. Pack. Tech., 25:495-505, 2002. doi:10.1109/TCAPT.2002.803653 C292

[8] O. Erdinc, B. Vural and M. Uzunoglu. A dynamic lithium-ion battery model considering the effects of temperature and capacity fading. IEEE Inter. Conf. Clean Elec. Power, 383-386, 2009. doi:10.1109/ICCEP.2009.5212025 C292

[9] B. Schweighofer, K. M. Raab and G. Brasseur. Modeling of high power automotive batteries by the use of an automated test system. IEEE T. Instrum. Meas., 52:1087-1091, 2003. doi:10.1109/TIM.2003.814827 C292

[10] S.-Y. Lee, W.-L. Chiu, Y.-S. Liao, K.-Y. Lee, J.-H. Chen, H.-J. Lin and K. Li. Modified empirical fitting of the discharge behavior of $\mathrm{LiFePO}_{4}$ batteries under various conditions. ANZIAM J., 55:368-383, 2014. doi:10.21914/anziamj.v55i0.8182 C293, C295, C297

[11] S. Flandrois and B. Simon. Carbon materials for lithium-ion rechargeable batteries. Carbon, 37:165-180, 1999. doi:10.1016/S0008-6223(98)00290-5 C294

[12] H. Li and H. Zhou. Enhancing the performances of Li-ion batteries by carbon-coating: present and future. Chem. Comm., 48:1201-1217, 2012. doi:10.1039/C1CC14764A C294 
[13] J. Wang and X. Sun. Understanding and recent development of carbon coating on $\mathrm{LiFePO}_{4}$ cathode materials for lithium-ion batteries. Energ. Environ. Sci., 5:5163-5185, 2012. doi:10.1039/C1EE01263K C294

[14] P. Bai and M. Z. Bazant. Charge transfer kinetics at the solid-solid interface in porous electrodes. Nature Comm., 5:3585, 2014. doi:10.1038/ncomms4585 C294

\section{Author addresses}

1. Chi-Yao Chung, Department of Engineering Science and Ocean Engineering, National Taiwan University, Taipei 10617, Taiwan. mailto:r03525049@ntu.edu.tw

2. Kung-Yen Lee, Department of Engineering Science and Ocean Engineering, National Taiwan University, Taipei 10617, Taiwan. mailto:kylee@ntu.edu.tw

3. Ting-Jung Kuo, Department of Engineering Science and Ocean Engineering, National Taiwan University, Taipei 10617, Taiwan. mailto:tingjungk@ntu.edu.tw

4. Zih-Yeh Lin, Department of Engineering Science and Ocean Engineering, National Taiwan University, Taipei 10617, Taiwan. mailto:r02525045@ntu.edu.tw

5. Shuen-De Wu, Department of Mechatronic Engineering, National Taiwan Normal University, Taipei 10610, Taiwan. mailto:sdwu@ntnu.edu.tw

6. Chiao Fu, Department of Engineering Science and Ocean Engineering, National Taiwan University, Taipei 10617, Taiwan. mailto:r04525045@ntu.edu.tw 